\title{
Shear-induced microstructural changes in flocculated suspensions of fumed silica
}

\author{
Srinivasa R. Raghavan and Saad A. Khan ${ }^{\text {a) }}$ \\ Department of Chemical Engineering, North Carolina State University, \\ Raleigh, North Carolina 27695-7905
}

(Received 10 April 1995; accepted 19 July 1995)

\begin{abstract}
Synopsis
Suspensions of fumed silica exhibit a wide range of rheological properties, depending on the type of microstructure present. At high silica concentrations, the rheological behavior is "gel-like," due to the formation of a network consisting of interconnected silica flocs. When large amplitude oscillatory preshear is applied on these systems, the network linkages are disrupted, resulting in the formation of isolated focs. In this study, we focus on the extent to which the network is restored on cessation of preshear. By applying small amplitude oscillations we can study the development of the elastic modulus $\left(G^{\prime}\right)$ with time, following disruptive shear. We find that the restoration of the network after preshear is instantaneous; however, $G^{\prime}$ recovers to different levels depending on the amplitude of the imposed preshear strain. Contrary to expectations, larger preshear strains (which cause a greater degree of microstructural disruption) do not always lead to lower levels of recovered $G^{\prime}$. For strains greater than a critical value, the recovered $G^{\prime}$ progressively increases with increasing preshear strain. This anomalous behavior of the elastic modulus is explained in terms of microstructural rearrangements that may occur during oscillatory preshear. (c) 1995 Society of Rheology.
\end{abstract}

\section{INTRODUCTION}

Fumed silica is an extremely versatile material that can be used to modify the rheology of a variety of systems. It has been widely employed as a filler in silicon rubber-based products and the rheological properties of such systems have been well documented (Ziegelbaur and Caruthers, 1985; Aranguren et al., 1992; Cochrane and Lin, 1992). In addition, fumed silica can function as an effective thickening and thixotropic agent in low molecular weight solvents. Several novel applications are being found for these suspensions. For example, gels of fumed silica in mineral or silicone oils are used as filling compounds in fiber-optic cables (Khan et al., 1991). Suspensions of fumed silica in polyethylene glycols are being considered for application as polymer electrolytes in rechargeable lithium batters (Khan et al., 1994).

Fumed silica is prepared by the flame hydrolysis of silicon tetrachloride $\left(\mathrm{SiCl}_{4}\right)$ in a flame of $\mathrm{H}_{2}$ and $\mathrm{O}_{2}$ (Degussa Tech. Bull., 1989). Initially, spherical particles of silica $(\sim 7-40 \mathrm{~nm}$ diameter $)$ are formed, and these fuse irreversibly into aggregates. Silanol groups $(\mathrm{Si}-\mathrm{OH})$ are generated on the silica surface during the preparation process. When dispersed in liquids, adjacent aggregates can interact via silanol-silanol hydrogen bonding, giving rise to larger structures called flocs. At high silica concentrations a three-

${ }^{\text {a) }}$ Corresponding author.

(c) 1995 by The Society of Rheology, Inc.

J. Rheol. 39(6), November/December 1995

0148-6055/95/39(6)/1311/15/\$7.00 
dimensional network of flocs extends throughout the volume of the sample and the suspension is considered to be a "physical gel" (Kosinski and Caruthers, 1985; Khan and Zoeller, 1993). The flocculated microstructure thus has multiple levels: The aggregates can be regarded as the primary structure of the silica, the flocs are made up of interconnected aggregates, and the network is composed of flocs.

The rheological properties of fumed silica suspensions are influenced by several factors: the concentration of the silica, the polarity of the solvent, and the surface chemistry of the silica. The effects of these variables were investigated for suspensions in oligomeric liquids by Khan and Zoeller (1993), using dynamic rheology and light scattering. For suspensions in mineral oil, the elastic modulus $G^{\prime}$ scaled with the particle volume fraction $\phi$ according to the relation $G^{\prime} \propto \phi^{4}$. In addition, the silica flocs in mineral oil were found to possess a fractal nature with a fractal dimension $D=1.75$. The rheological experiments reported in this study were confined to the linear viscoelastic region of the samples. The application of large dynamic strains to such systems results in deviations from linearity, owing to the disruption of the sample-spanning network structure. The present study is primarily concerned with the effect of disruptive shear on the rheology and microstructure of fumed silica suspensions.

Transient structures existing in fumed silica suspensions in polydimethyl siloxane (PDMS) melts have been studied by Caruthers and co-workers, using steady shear techniques (Ziegelbaur and Caruthers, 1985; Kosinski and Caruthers, 1985). They measured the stress growth function, following a high rate of preshear and a rest period. The preshear disrupted the microstructure which was restored to different extents depending on the rest time. For relatively large rest times, the stress growth function exhibited an overshoot, which was attributed to the presence of a network-type microstructre (Kosinski and Caruthers, 1985).

Mewis and co-workers (Mewis and de Bleyser, 1972; Schoukens and Mewis, 1978) have demonstrate the inherent disadvantages in using steady shear methods to characterize transient structures. In steady shear techniques the viscosity at a given rate of shear is used to characterize unambiguously the instantaneous structure. This assumption is valid only if the material is inelastic; it is inapplicable for viscoelastic suspensions. Moreover, in all steady shear measurements, the system has to be disturbed in the process of estimating the extent of recovery. Thus the structure being examined gets disrupted owing to the measurements themselves.

Oscillatory shear measurements, however, are nonintrusive, provided they are carried out in the linear viscoelastic region of the material. Mewis and de Bleyer (1972) used this method to investigate structural growth in suspensions of a polyamide gel in linseed oil. The initial network structure was destroyed by means of steady preshear at a large rate. Upon cessation of preshear, oscillatory measurements in the linear region were conducted to probe the structural recovery. The elastic modulus was found to increase with time, starting from an initial value $G_{0}^{\prime}$. The level of $G_{0}^{\prime}$ was lower, following a higher preshear rate, indicating that a greater degree of structural breakdown was caused by larger preshear. The authors also showed that the network structure was restored almost instantaneously on cessation of preshear. The network continued to develop with time, thus explaining the increase in elastic modulus. Similar results were reported with suspensions of carbon black in mineral oil (Schoukens and Mewis, 1978).

Structural breakdown can also be induced by imposing oscillatory shear at large strain amplitudes outside the linear regime. Kanai et al. (1992) presheared their suspensions of ferric oxide particles in mineral oil using oscillations of $100 \%$ strain amplitude and 50 $\mathrm{rad} / \mathrm{s}$ frequency. During this period of intense shearing, the initial network present in the sample was broken down, and the rheological response was determined by isolated flocs. 
Network development following preshear was monitored by measurements of $G^{\prime}$ at low strain and frequency. The postshear $G^{\prime}$ increased slightly with time until it reached a plateau. The results indicated that a network was formed immediately after preshear and this structure strengthened as time progressed. The authors were able to explain their findings using a site-percolation model.

In the present study, we systematically investigate the effects of large amplitude oscillatory shear on the rheology and microstructure of fumed silica suspensions. In order to study structural development on cessation of large shear, dynamic measurements are conducted at a low strain within the linear regime of the suspension. An understanding of shear-induced structural changes is critical from a fundamental standpoint. These aspects are also industrially important, because high levels of shear are typically encountered in many applications.

\section{EXPERIMENT: MATERIALS AND METHODS}

We have used two different fumed silicas, Aerosil D150 and R805 (Degussa Corporation, Allendale, NJ) for our study. D150 is a typical hydrophilic fumed silica, with silanol groups present on its surface. R805 is a partially hydrophobic variety, prepared by replacing half of the surface silanols with $n$-octyl $\left(-\mathrm{C}_{8} \mathrm{H}_{17}\right)$ groups (Degussa Tech. Bull., 1990). Both silicas have approximately the same surface area $\left(150 \mathrm{~m}^{2} / \mathrm{g}\right)$. The $R 805$ silica-silica interactions in a liquid medium may involve both the hydrogen bonding interactions of silanols and also secondary dispersive interactions between the alkyl chains (Khan and Zoeller, 1993). Due to the decreased surface silanol density, the degree of network connectivity in a nonpolar medium is generally lower with R805 than with a corresponding hydrophilic silica of similar surface area. Alkyl-alkyl dispersive interactions predominate in polar media owing to competitive hydrogen bonding of the silanols with molecules of the continuous phase.

The liquid media used in our study were a mineral oil and a polypropylene glycol (PPG) (Aldrich Chemicals). The mineral oil consisted of mixed alkane hydrocarbons (average $\mathrm{C}_{23}$ ) and had a viscosity at ambient temperature of about $25 \mathrm{cP}$. The PPG had a molecular weight of 725 and exhibited an unfilled viscosity of approximately $120 \mathrm{cP}$.

Each suspension was prepared by adding the liquid to the silica in a blender and mixing for approximately $1 \mathrm{~min}$. This had been determined earlier to be the minimum mixing time required to ensure reproducibility between batches (Khan and Zoeller, 1993). Samples were made in batches of $60 \mathrm{ml}$ and placed under vacuum at room temperature for about $12 \mathrm{~h}$ to remove air bubbles. The concentration of silica used was $5 \%$ in mineral oil and $10 \%$ in PPG (w/w).

Dynamic rheological experiments were carried out using a Rheometrics Mechanical Spectrometer (RMS-800) in a cone-and-plate geometry. The results were checked on two different cone-and-plate fixtures - one having a cone angle of $0.1 \mathrm{rad}$ and a diameter of $25 \mathrm{~mm}$ and the other having a cone angle of $0.04 \mathrm{rad}$ and a diameter of $50 \mathrm{~mm}$. In all cases, the samples were allowed to rest for about $10 \mathrm{~min}$ after loading before any measurements were taken. The same mixing, spreading, and loading techniques were used for all samples. The data reported here were reproducible with a relative error of less than $10 \%$.

\section{RESULTS}

\section{A. Nonlinear viscoelastic behavior}

Our initial efforts focused on examining the viscoelastic behavior of fumed silica suspensions at large strain amplitudes. The experiments described henceforth were all 


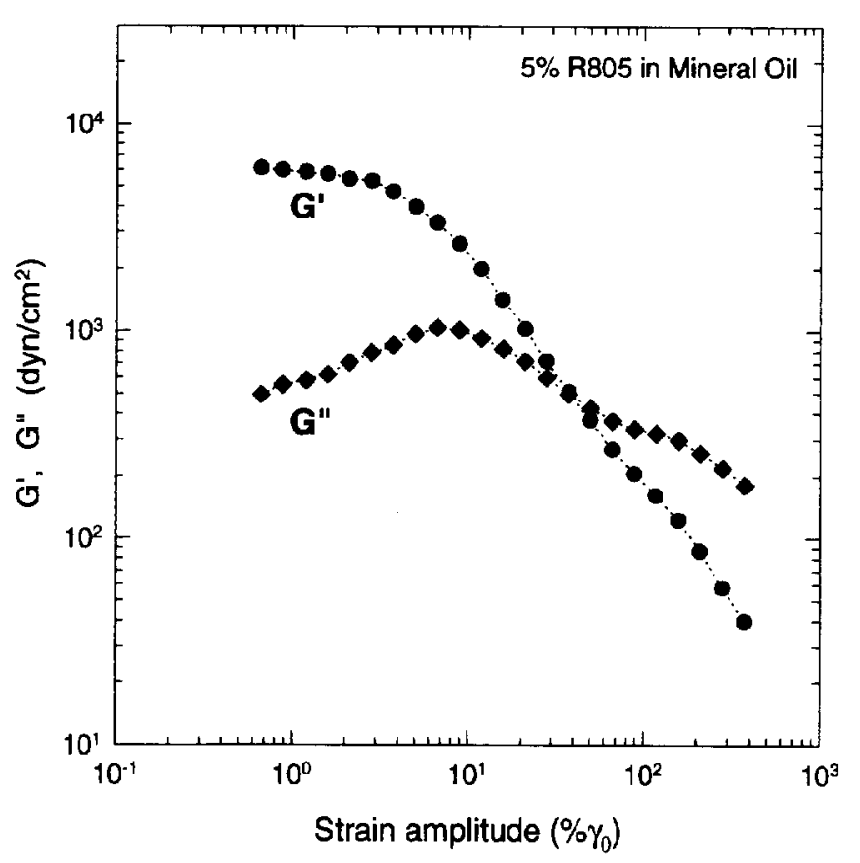

FIG. 1. Dependence of the elastic modulus $\left(G^{\prime}\right)$ and viscous modulus $\left(G^{\prime \prime}\right)$ on strain amplitude $\left(\% \gamma_{0}\right)$. This strain sweep experiment was conducted at a constant frequency of $10 \mathrm{rad} / \mathrm{s}$.

conducted on a suspension containing $5 \%(\mathrm{w} / \mathrm{w})$ of R805 hydrophobic silica in mineral oil. In Fig. 1, we show the results of a typical strain sweep experiment conducted on a sample, after loading. The elastic modulus $G^{\prime}$ decreases rapidly with increasing strain amplitude $\left(\% \gamma_{0}\right)$, for strains outside the linear viscoelastic regime. The viscous modulus $G^{\prime \prime}$ shows a maximum at a moderate strain amplitude (aboul 8\%) and is higher than $G^{\prime}$ at very large strains. Within the linear range, the moduli are largely independent of $\gamma_{0}$. The above behavior indicates the destruction of structure due to oscillatory shear at large strain amplitudes. Under large deformations the sample-spanning network ceases to exist and isolated flocs are formed. It should be noted that when $G^{\prime}$ and $G^{\prime \prime}$ are no longer in the linear region, the values reported can only be regarded as apparent values. Nevertheless, the strain sweep illustrates the general trends in variation of moduli, and hence structure, with strain amplitude.

The increased structural disruption at higher imposed strains can be demonstrated in a clearer fashion by shearing the samplc at a constant strain amplitude and frequency. The results of several such "time sweeps," each at a different strain amplitude, and a constant frequency of $10 \mathrm{rad} / \mathrm{s}$, are shown in Fig. 2. For a time sweep at $2 \%$ strain (within the linear regime), $G^{\prime}$ increases slightly with time. The other curves show that when the sample is sheared at larger strains, $G^{\prime}$ drops down to lower levels. The decrease in $G^{\prime}$ with time is small, suggesting that most of the structural disruption occurs immediately after the deformation is applied.

\section{B. Structure recovery after oscillatory preshear}

Our investigation of structural breakdown caused by large amplitude strains and subsequent structural regrowth was performed using a prescribed sequence, consisting of 


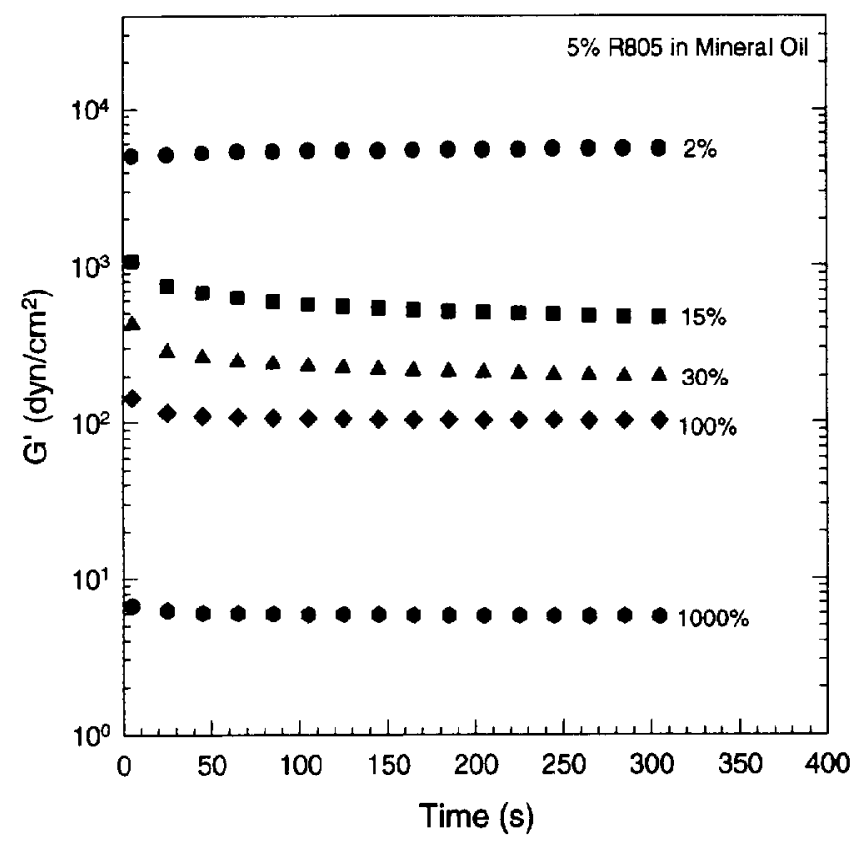

FIG. 2. Elastic modulus as a function of time for different strain amplitudes. The strain amplitude in these time sweeps is indicated beside each curve. The frequency of the oscillations was kept constant at $10 \mathrm{rad} / \mathrm{s}$ in all cases.

time sweeps done in succession. Initially, a "calibration" experiment was performed at $2 \%$ strain for $1 \mathrm{~min}$, to ensure that the sample was in a reproducible state before the application of large shear. Following this, a constant (large) strain amplitude was imposed on the sample for $5 \mathrm{~min}$, to produce structural disruption. For simplicity, we shall refer to this as the preshear experiment. The next time sweep was started immediately on cessation of preshear (there is a gap of about $12 \mathrm{~s}$ before the new experiment starts) and was carried out at the same low strain of $2 \%$, as in the initial calibration. The purpose of this experiment is to study the recovery of structure after cessation of large shear, and it will therefore be called the "recovery" or "growth" sweep. We performed several such experimental sequences on our suspension, each with a different strain amplitude in the preshear. A new sample was loaded into the rheometer for each sequence.

In Fig. 3, we show the structural regrowth in terms of the developing elastic modulus, following preshear at two different strain amplitudes: 30\% [Fig. 3(a)] and 1000\% [Fig. 3(b)]. The initial level of modulus prior to the preshear is the same in each case, as indicated by the dashed lines. We consider the values of $G^{\prime}$ during the growth sweeps to be characteristic of the structure present in the suspension during that period. For this to be true, the low amplitude (2\%) oscillations must not damage the structure as the measurements are being made. The validity of this assumption will he demonstrated subsequently. For the moment, we will examine the significance of the data in Fig. 3.

In Fig. 3(a), a strain amplitude of $30 \%$ is applied during preshear. Following this, the level of $G^{\prime}$ in the growth sweep, henceforth called $G^{\prime}$ (recovered), is about 1500 dyn $/ \mathrm{cm}^{2}$, in comparison with the initial modulus of about $6000 \mathrm{dyn} / \mathrm{cm}^{2}$. In Fig. 3(b), a 


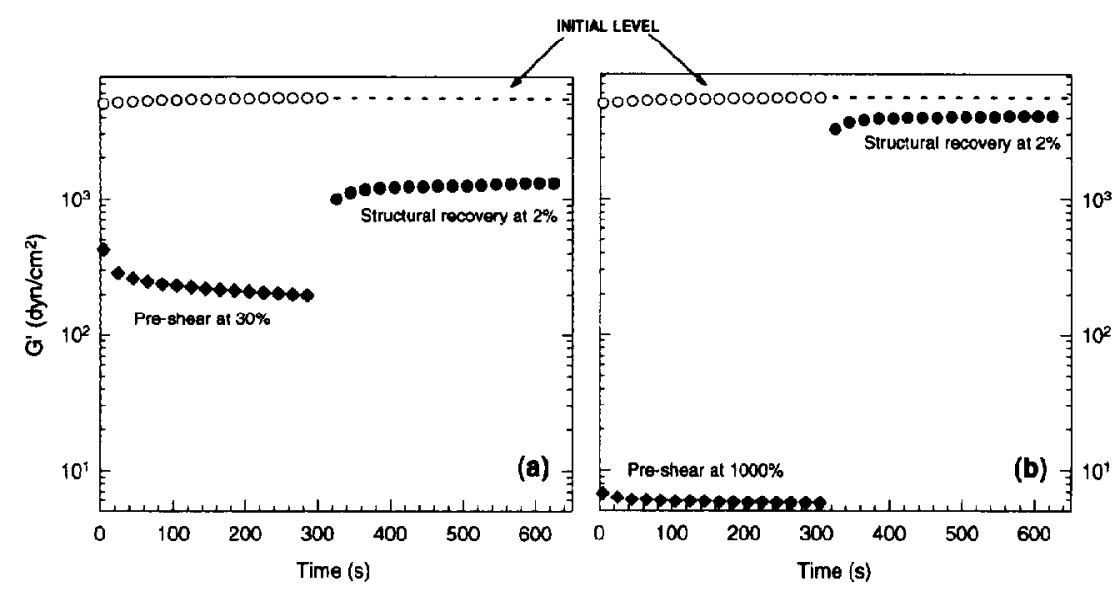

FIG. 3. Structural breakdown due to large amplitude preshear followed by structural recovery at low (2\%) strain, monitored in terms of the elastic modulus $G^{\prime}$. (a) corresponds to preshear at $30 \% \gamma_{0}$ while (b) corresponds to preshear at $1000 \% \quad \gamma_{0}$. All experiments were performed at a frequency of $10 \mathrm{rad} / \mathrm{s}$. The sample is $5 \%$ R805 fumed silica in mineral ail as before.

larger preshear strain $(1000 \%)$ is applied, which results in a lower level of the apparent $G^{\prime}$ during this process. However, the level of $G^{\prime}$ (recovered) is significantly higher, at about 4000 dyn $/ \mathrm{cm}^{2}$.

In Figs. 4(a) and 4(b), we show growth sweeps following different levels of large amplitude deformation. The numbers adjacent to the curves represent the strain amplitude in the preshear. Each of these curves represents a different experiment performed on a fresh sample. These data provide information on the recovery of structural elasticity after various shear histories. For a range of small preshear strains (up to $\sim 30 \%$ ), the level of $G^{\prime}$ (recovered) decreases with increasing strain. However, for strains larger than $30 \%$ [i.e., 50\%-1000\% in Fig. 4(b)], the recovery is greater after a larger strain amplitude. Since the microstructure is increasingly broken down by larger preshear strains, as demonstrated in Figs. 1 and 2, the enhanced recovery observed after large deformations seems anomalous.

The relationship between the recovered modulus and the preshear strain can be better observed in Fig. 5 where we have cross-plotted the two variables. The values of $G^{\prime}$ (recovered) were obtained from the growth sweeps in Fig. 4, at a time of 1 min, after cessation of large amplitude preshear. This plot shows a characteristic minimum, $\gamma_{0}^{\min }$ at about $30 \%$ strain. Thereafter, $G^{\prime}$ (recovered) increases until it levels off. The structural recovery phenomenon seems to follow two ranges of distinct behavior: the first, for strains below $\gamma_{0}^{\min }$ during which the recovered elasticity follows expected trends with increasing shear, and the second, for strains above $\gamma_{0}^{\min }$ for which the trends are quite unusual.

To better understand the recovery behavior, we probed the microstructure by performing frequency sweeps (at $2 \%$ strain) after the termination of preshear. The results of such sweeps done after oscillatory preshear at $30 \%$ and $1000 \%$ strains are shown in Figs. 6(b) and $6(\mathrm{c})$, respectively. Figure $6(\mathrm{a})$ is a frequency sweep conducted before preshear where we find the initial $G^{\prime}$ and $G^{\prime \prime}$ to be largely independent of frequency as expected. A frequency independent plateau for $G^{\prime}$ indicates a gel-like character and the presence of a network structure. Strikingly, a similar broad plateau for $G^{\prime}$ can be observed in Figs. 6 (b) and $6(c)$ as well. Since these frequency sweeps were done immediately after cessation of 

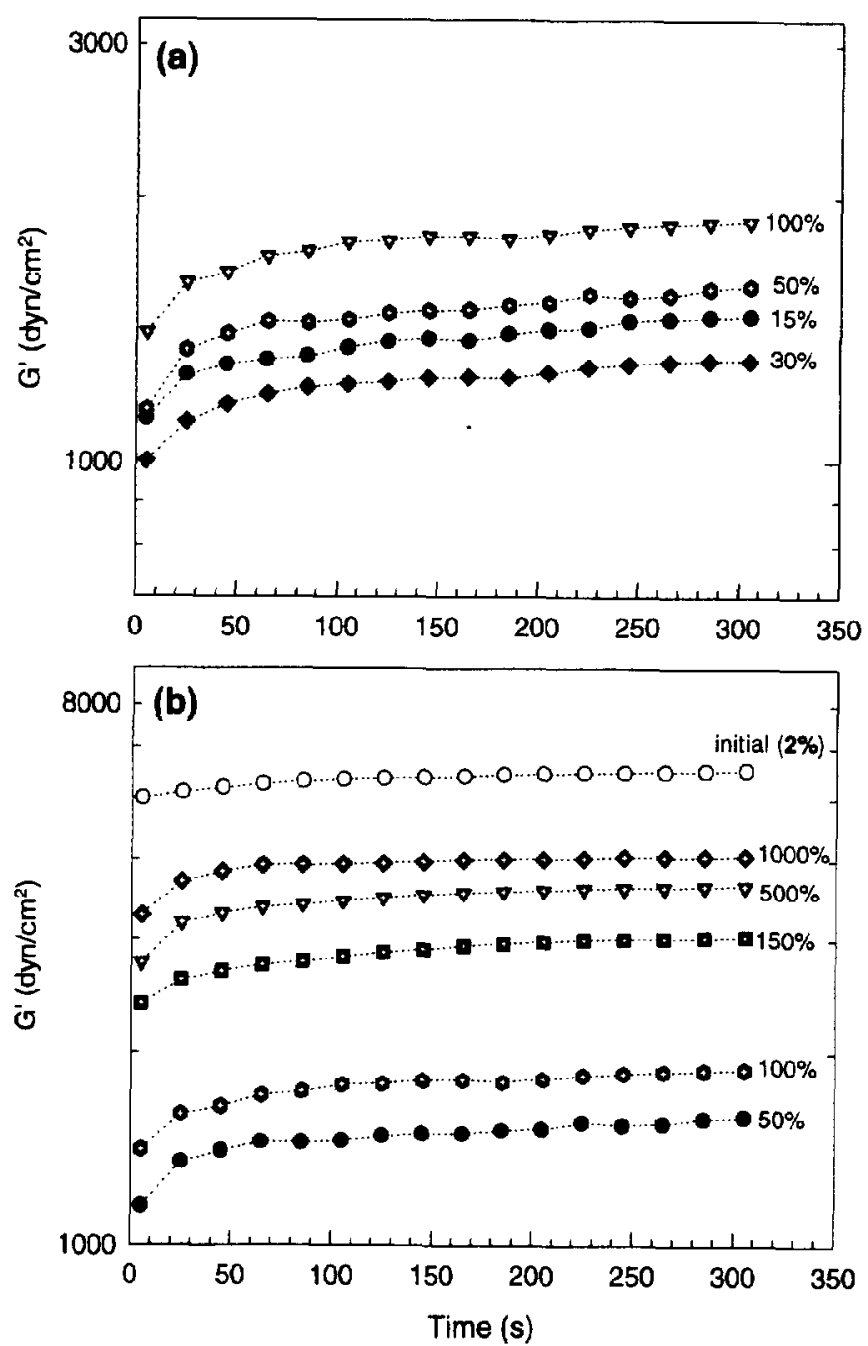

FIG. 4. Growth (recovery) of the elastic modulus after preshear at several strain amplitudes. The strain amplitude of preshear is indicaled beside each curve. All experiments were conducted at $10 \mathrm{rad} / \mathrm{s}$ frequency.

disruptive shear, it implies that a network structure is reestablished almost instantaneously. This is also corroborated by the fact that the growth sweeps in Fig. 4 do not show a significant increase with time, which would be expected if gelation (network formation) occurred at a finite time after cessation of preshear (Khan et al., 1989).

The immediate establishment of network after cessation of disruptive preshear has been observed for flocculated suspensions (Mewis and de Bleyser, 1972; Kanai et al., 1992; Kanai and Amari, 1993). Due to the imposed preshear, a certain distribution of isolated flocs will be produced. On cessation of preshear, a network can be established through connections between adjacent flocs. If the interfloc attraction is sufficiently strong, the network formation can occur instantaneously as preshear is terminated. Thus the flocs present during the preshear stage will form the backbone of the network that will exist subsequently (Kanai and Amari, 1993). 


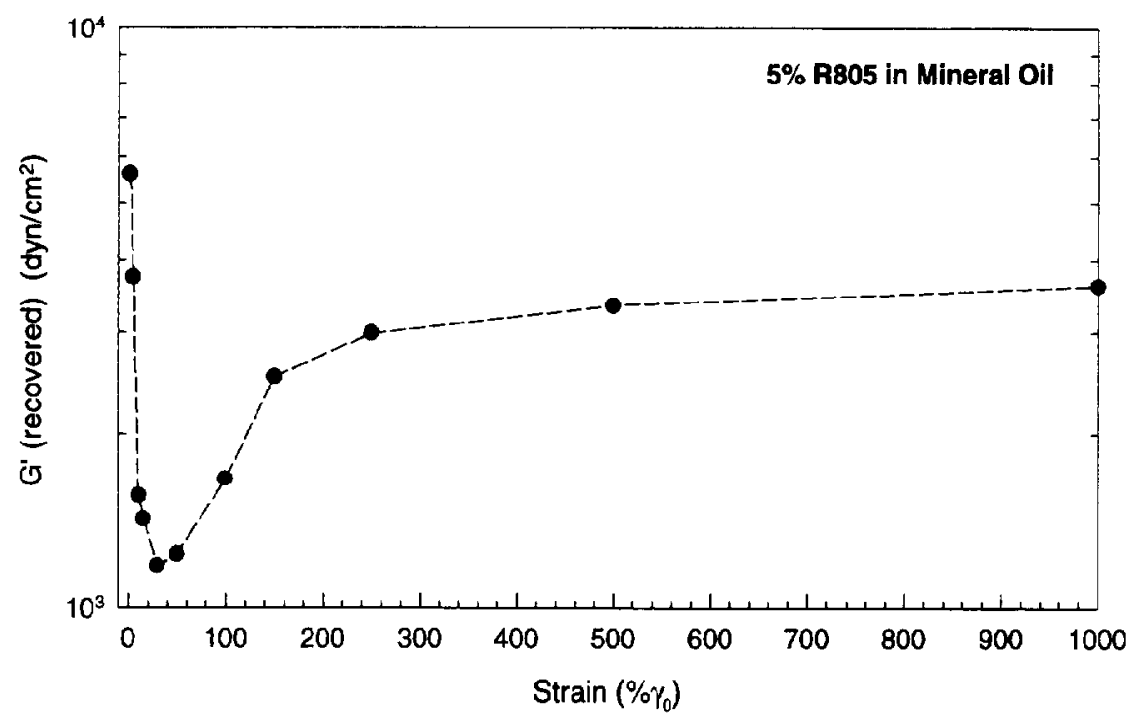

FIG. 5. Plot of $G^{\prime}$ (recovered) as a function of the strain amplitude of preshear. $G^{\prime}$ values were obtained from the recovery (growth) sweeps, as shown in Fig. 4, after a time interval of 1 min. All the data correspond to a frequency of $10 \mathrm{rad} / \mathrm{s}$.

The flat shape of the elastic modulus in Fig. 6 also suggests that the frequency at which we monitor the structural growth is not a critical factor. It should be mentioned that the strain dependence of the recovering structures was determined by performing strain sweeps after various extents of preshear. The onset of nonlinearity occurred at strains grcater than $2 \%$ in all cases. This justifies the choice of a $2 \%$ strain for the growth sweeps as well as the frequency sweeps, since that is within the linear range of the developing structures.

\section{Time-dependent aspects of structural growth}

We have assumed that the low amplitude $(2 \%)$ oscillations imposed during the growth sweep did not hinder structural growth. To test this, we studied the recovery process in
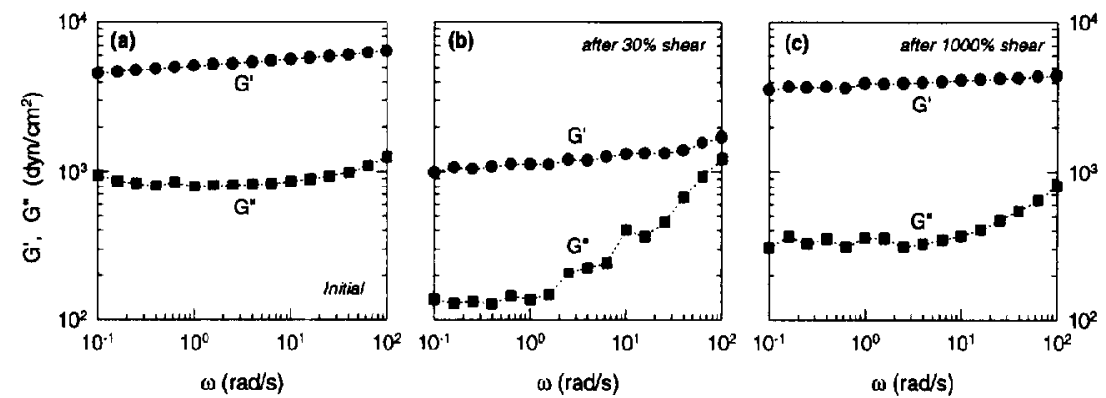

FIG. 6. Elastic and viscous moduli as a function of frequency. All experiments were done at a strain amplitude of $2 \%$. (a) corresponds to the initial sample. (b) and (c) represent data for samples that have been exposed to 5 min of preshear at $30 \%$ and $1000 \%$ strain amplitudes, respectively. 


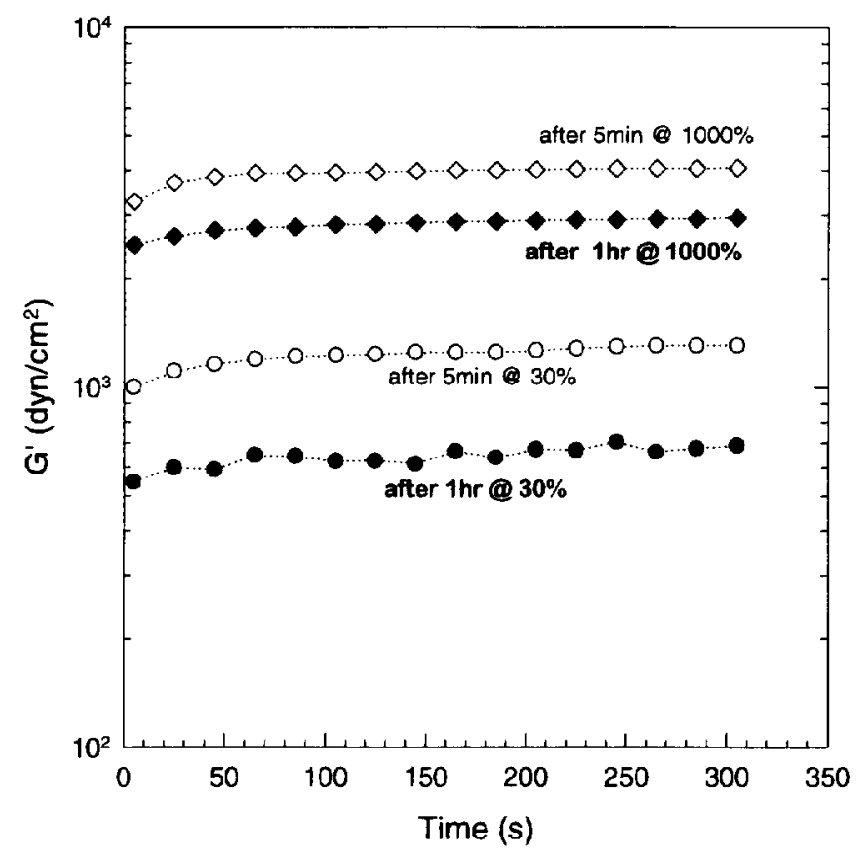

FIG. 8. Dependence of the recovery (growth) of the elastic modulus on the time of preshear: The open symbols correspond to 5 min preshear whereas the filled symbols correspond to $1 \mathrm{~h}$ of preshear.

two different ways - the first being a growth sweep in the continuous mode, identical to those described earlier, while the second involved intermittent measurements of $G^{\prime}$ at intervals of $10 \mathrm{~min}$ over a period of $1 \mathrm{~h}$. Data obtained by each method following a 30\% preshear are plotted in Fig. 7. The continuous measurement at $2 \%$ strain shows higher values for $G^{\prime}$ (recovered). The aim of performing these experiments was to compare structural recovery at rest with that under a constant low amplitude shear. Our data indicates that the application of continuous oscillations leads to faster recovery. Nevertheless, the data from the two experiments are only marginally different. Therefore, the applicability of this method to detect structural changes seems to be validated.

The experiments shown in Fig. 7 were performed for a period of $1 \mathrm{~h}$. It can be observed that the increase in $G^{\prime}$ over this period is not very large, relative to its initial level at $t=0$, i.e., immediately after preshear. For this reason, we have not performed a detailed study on the kinetics of growth. The increase in $G^{\prime}$ arises from the development of the network structure that was initially formed, and this seems to be promoted by the action of the small oscillations. However, once a network is established, its essential features will remain largely unaltered even after a long time, since large scale reorganization of the elements is physically prevented (Kosinski and Caruthers, 1985). This can explain why the extent of increase in $G^{\prime}$ is small.

We now illustrate the effect of preshearing for a time longer than $5 \mathrm{~min}$. To study this, we presheared samples for $1 \mathrm{~h}$ at strains of $30 \%$ and $1000 \%$, and then recorded the corresponding growth sweeps. These are displayed in Fig. 8 together with the growth sweeps obtained after 5 min preshear at each strain (shown earlier in Fig. 4). The level of $G^{\prime}$ (recovered) is observed to be lower, when preshear is carried out for a longer time. However, the recovery behavior of the system seems to remain unchanged - the increase 


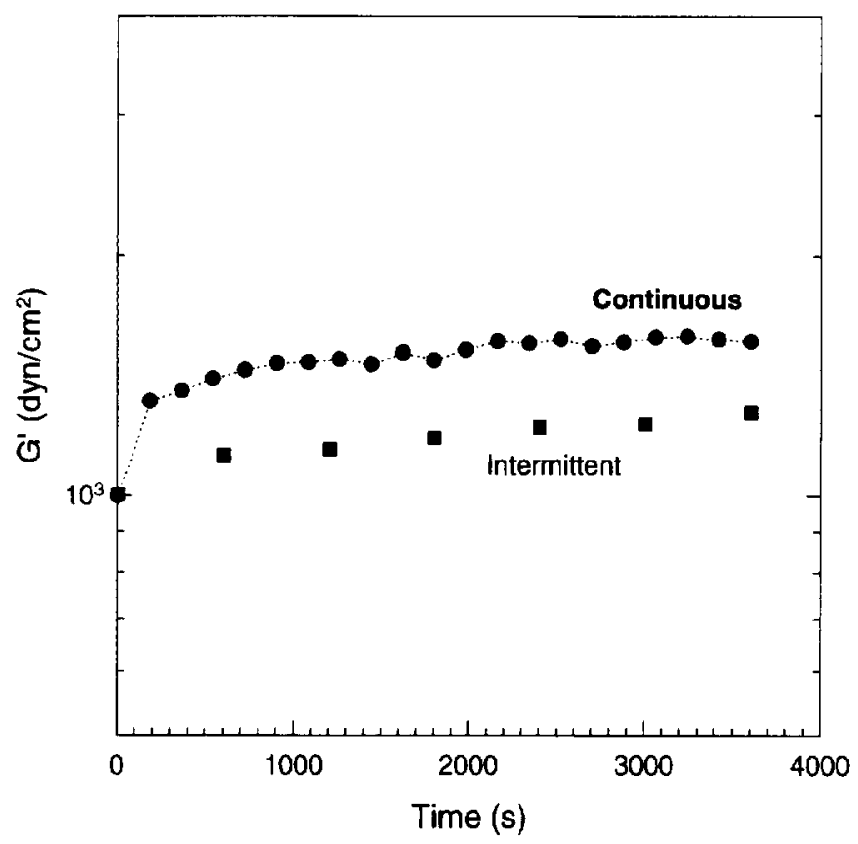

FIG. 7. Measurement of $G^{\prime}$ (recovered) after $30 \%$ preshear: Continuous measurements (continuous low amplitude shear at $2 \%$ strain, $10 \mathrm{rad} / \mathrm{s}$ ) are compared with intermittent measurements made every 10 min at the same strain and frequency.

of $G^{\prime}$ (recovered) with time is approximately the same, and $G^{\prime}$ (recovered) is higher after $1000 \%$ shear, in both cases.

Our study of the time-dependent aspects of structural recovery reveals that it is not possible to precisely quantify the value of $G^{\prime}$ (recovered). This value depends both on the time into the growth sweep as well as the time for which breaking shear is performed. Nevertheless, we observe that the recovery behavior is primarily dictated by the strain history-i.e., the large amplitude strain that is applied on the sample during preshear. The effects of the other variables, viz., frequency and time, are comparatively small.

\section{Other preshear histories}

We also subjected the suspension to more complex strain histories involving different nonlinear strains. In Fig. 9, two strain histories are compared: in (A), 30\% preshear is applied for $10 \mathrm{~min}$; in (B), 30\% preshear is performed for $5 \mathrm{~min}$, followed by $5 \mathrm{~min}$ of $1000 \%$ preshear. Values of $G^{\prime}$ are shown throughout, with the final experiment in each case performed at $2 \%$ strain to detect structural recovery. The recovered $G^{\prime}$ after strain history (B) is much higher than after (A). This shows that the elastic structure of the suspension following moderate shear can be enhanced by subjecting it to more intense oscillatory shear. Similar findings were reported in a recent study on flocculated suspensions of ferric oxide in mineral oil (Kanai and Amari, 1993). The authors also observed "strain-shear thickening" in their suspensions-i.e., a range of strains within a strain sweep experiment, for which the modulus increased with increasing strain amplitude. Analogous experiments on our samples did not yield similar results; the decrease in $G^{\prime}$ 

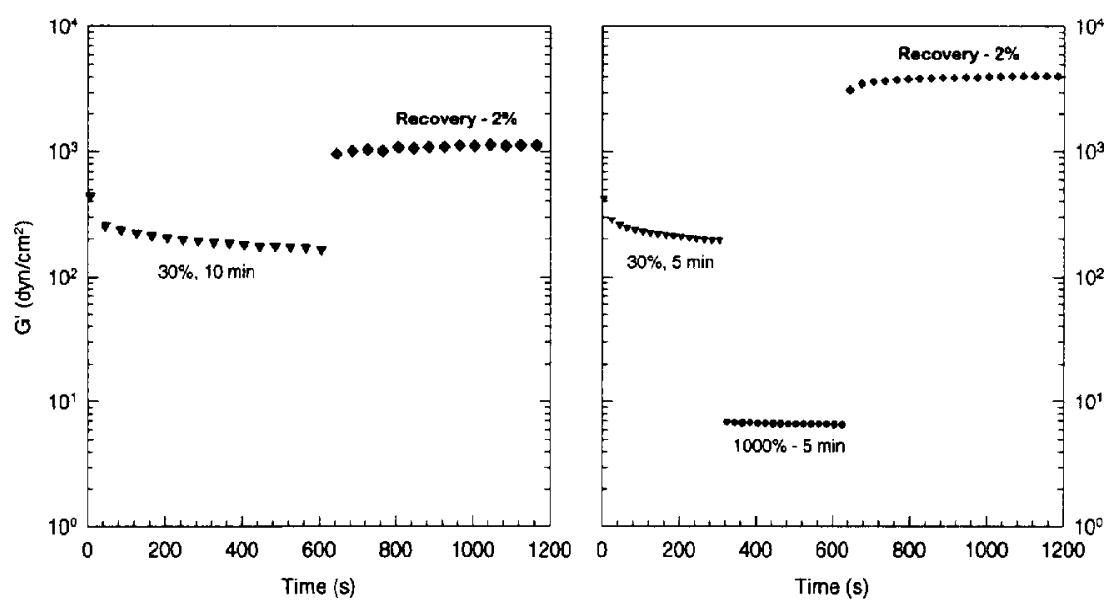

FIG. 9. Development of elastic modulus (at $2 \% \gamma_{0}$ ) after different strain histories: (a) corresponds to $30 \%$ shear for $10 \mathrm{~min}$; Fig. 9 (b) corresponds to $30 \%$ shear for $5 \mathrm{~min}$, followed by $1000 \%$ shear for $5 \mathrm{~min}$.

with increasing strain amplitude was monotonic, as shown in Fig. 1. The phenomenon that we have described, theretore, cannot be interpreted as strain thickening.

We also performed experiments using steady preshear instead of large dynamic strains. The preshear was done at a constant shear rate for $5 \mathrm{~min}$ and was followed by oscillatory growth sweeps at $2 \%$ strain as before. Interestingly, as Fig. 10 shows, we did not observe similar trends for $G^{\prime}$ (recovered) with preshear rates as we did with oscillatory strains. Instead, the level of $G^{\prime}$ (recovered) was approximately the same for a broad range of preshear rates from 0.001 to $100 \mathrm{~s}^{-1}$. For comparison we have shown the level of recovered modulus after oscillatory preshear at $1000 \%$ and $30 \%$ strains: The level of $G^{\prime}$ (recovered) is significantly lower following $30 \%$ oscillatory shear. Thus the anomalous recovery behavior is observed only with oscillatory preshear.

Recently, de Rooij (1994) found that the elastic modulus $G^{\prime}$ for weakly flocculated polystyrene latex dispersions is sensitive to the steady preshear rate in an unusual manner. For example, $G^{\prime}$ was observed to be lower following preshear at $0.12 \mathrm{~s}^{-1}$ than after preshear at $92 \mathrm{~s}^{-1}$. However, the phenomenon was not discussed in detail, and no oscillatory preshear data were reported. It is not possible, therefore, to compare his findings with ours. Nevertheless, it is of interest to determine whether different flocculated systems will exhibit the same peculiar behavior.

\section{E. Recovery behavior of different fumed silica suspensions}

We have obtained data similar to Figs. 1-10 for other fumed silica suspension systems. We present data for $G^{\prime}$ (recovered) as a function of preshear strain (Fig. 11) for two other suspensions: the first containing 5\% D150 silica in mineral oil and the second with $10 \%$ R805 silica in polypropylene glycol (PPG). Clearly, the two systems display the same anomalous recovery behavior described earlier. Each of these suspensions possesses a gel-like microstructure (Khan and Zoeller, 1993). A higher concentration of silica is required to form a gel in the polar PPG, since the glycol molecules are capable of hydrogen bonding with the silanol groups. The suspension of D150 in mineral oil has a very high initial modulus since the hydrophilic D150 has a greater silanol group density than R805. We have thus demonstrated that this unusual recovery phenomenon is not limited by the surface chemistry of the silica or the nature of the liquid medium. 


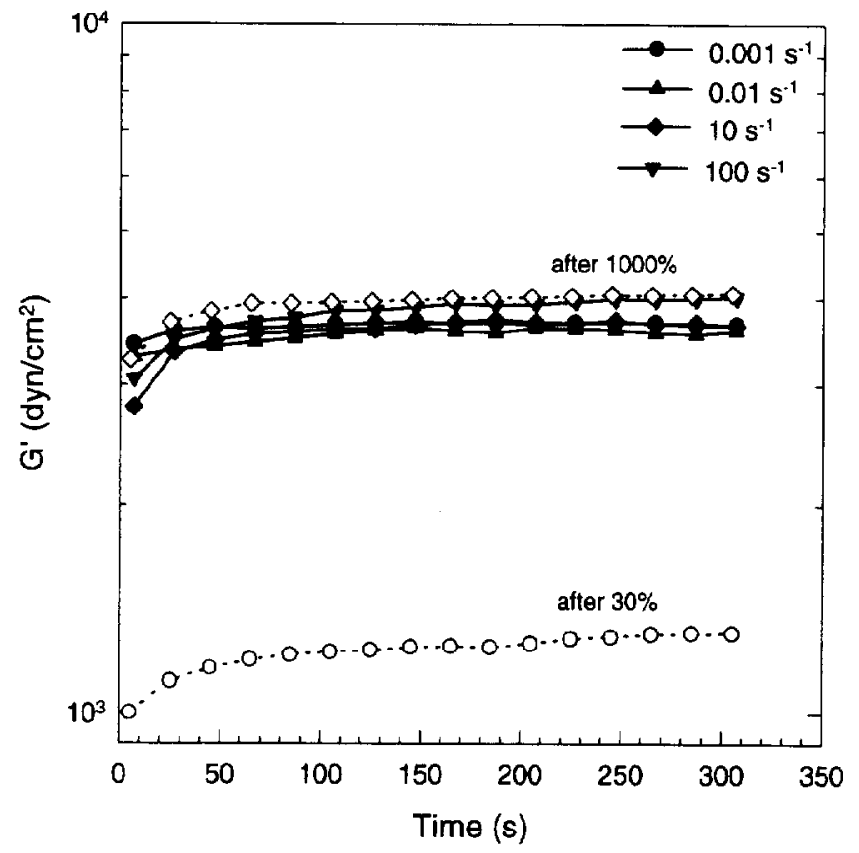

FIG. 10. Growth (recovery) of the elastic modulus after steady preshear at different shear rates. Recovery sweeps after oscillatory preshear at $1000 \%$ and $30 \%$ (open symbols) are also shown for comparison.

\section{DISCUSSION}

The most significant feature of our data is the unusual dependence of recovered $G^{\prime}$ on the strain amplitude of preshear, with the plot showing a characteristic minimum (Fig. 5). Evidently, the nature of the preshear dictates the features of the network structure present after preshear is ceased. We present in Fig. 12 a possible scenario of microstructural changes at low and high strain amplitudes, which could lead to the observed experimental results. The structure present initially in the sample (A) consists of small flocs, represented as branched chains, connected into a network. The bonds most susceptible to oscillatory shear will be those at the junctions between flocs. As a result, even a low strain amplitude shear outside the LVE range is sufficient to disrupt the network into fragments consisting of interconnected flocs. The average size of these fragments will decrease with increasing strain amplitude, as more bonds are disrupted.

At low strains $(\sim 15 \%)$, we postulate that the network fragments undergo rearrangement, resulting in the formation of larger flocs. In a thixotropic system, there is expected to be an equilibrium between bond breaking due to the action of shear forces and bond forming due to collisions between separated elements (Nguyen and Boger, 1985; Mewis, 1979). The tendency for bond formation through collisions would be significant at low strains, and would contribute to the formation of larger flocs. The net effect is to produce flocs which are composed of a larger number of aggregates than those present initially, as shown in (B). When shear is terminated, the network is formed by connections between the existing enlarged flocs. There would be fewer interfloc connections within the network structure when it is composed of larger flocs. This leads to a significantly lower $G^{\prime}$ as compared to the initial level. 


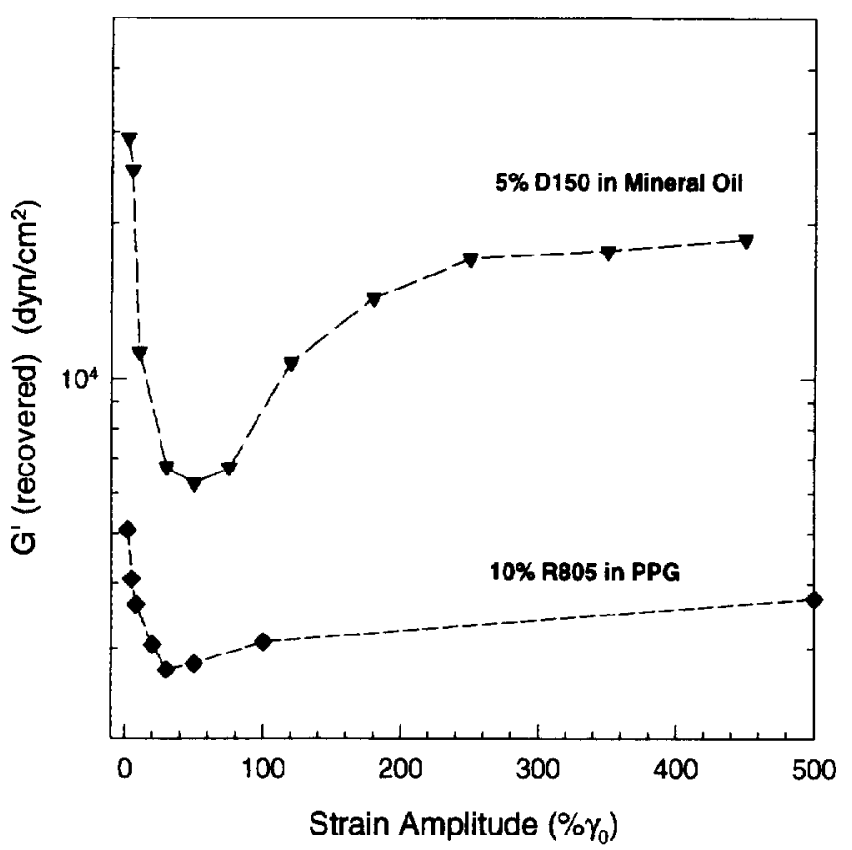

FIG. 11. Plot of $G^{\prime}$ (recovered) as a function of preshear strain amplitude. for two systems-5\% D150 silica in mineral oil and $10 \%$ R805 silica in PPG. $G^{\prime}$ values were obtained from recovery (growth) sweeps for each sample, similar to Fig. 4. The values plotted correspond to a time of $1 \mathrm{~min}$ into the growth sweep.

At very large strains, most of the network bonds would be disrupted, resulting in fragments of relatively small size. The equilibrium would be shifted overwhelmingly in favor of bond breaking, thereby minimizing the tendency to regroup into larger flocs. Thus the flocs eventually formed, will have sizes comparable to those initially present (C). When shear is ceased, the restored network is formed from smaller flocs. Due to a comparable number of physical crosslinks in the network, the restored elastic modulus is also comparable to initial levels. Our model thus makes use of the fact that smaller unit structures lead to a stronger network, due to an increase in the density of network connections. This has been theoretically predicted as well as experimentally observed (Van den Tempel, 1979).

The minimum in Fig. 5, corresponding to preshear at a critical strain amplitude $\gamma_{0}^{\min }$, may occur when the flocs produced by shear attain their largest sizes. It should be noted that at any strain amplitude there will be a competition between bond formation and bond disrpution. If bond formation is considered to occur due to collisions between separated elements, then it would be increasingly favored at larger strain amplitudes. However, the tendency for the bonds to get disrupted by the shear forces also increases with strain amplitude. Thus the occurrence of a minimum in the data may correspond to a shift in the balance between these two opposing tendencies.

The above qualitative reasoning can be extended to account for other experimental behavior reported, as well. The application of a large strain (1000\%) following preshear at a moderate strain (30\%) causes the disruption of the larger flocs formed during $30 \%$ shear into smaller structures. This increases the subsequent recovered modulus (Fig. 9). 


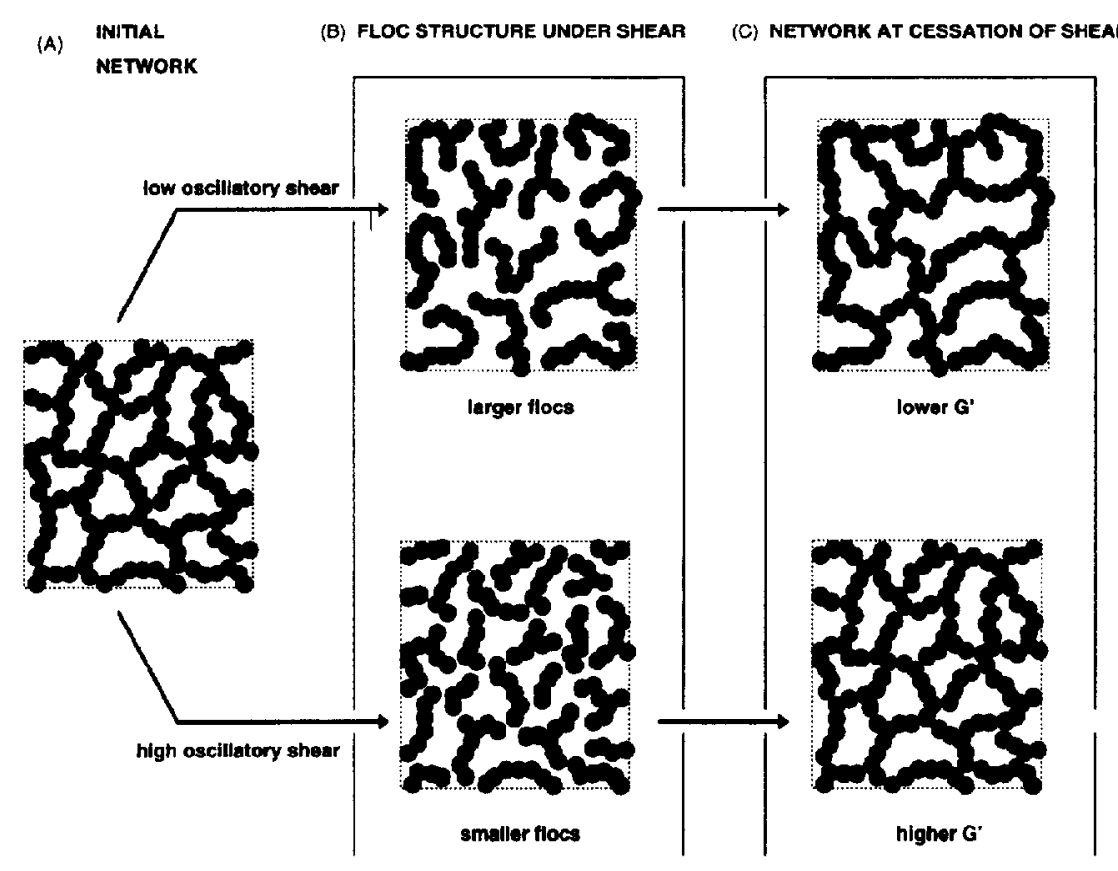

FIG. 12. Schematic representation of floc microstructure in fumed silica suspensions during and after oscillatory shear. (A) Initial floc structure; (B) floc structure under shear: The larger flocs under low shear are formed by structural rearrangement; (C) network formation at cessation of shear through connections between adjacent flocs. The restored network will have the same backbone for subsequent times.

The reason why the anomalous behavior is not observed following steady preshear could be because the microstructure is perturbed more under steady shear (Jones $e t a l$, 1991). The flocs may suffer significant displacement from their at-rest positions, in contrast to oscillatory shear, thus limiting the reorganization of structures. The effect of steady preshear is similar to that of large amplitude oscillatory preshear, and can be contrasted with the effect of moderate oscillatory shear (Fig. 10).

The above hypothesis is consistent with the unusual rheological behavior observed. However, there is no direct evidence in the literature for the structural changes postulated, though similar qualitative reasoning has been used in other cases (Kanai and Amari, 1993). Further investigations using techniques such as light scattering are necessary to elucidate the nature of these shear-dependent structures.

\section{CONCLUSIONS}

We have studied the effects of large amplitude oscillations on the rheology and microstructure of fumed silica suspensions. The initial microstructure in these suspensions is in the form of a space-filling network of flocs. When large amplitude oscillatory preshear is applied on these systems, the network is disrupted, and isolated flocs are formed. The recovery of structural elasticity after such destructive preshear can be characterized by the level of the restored elastic modulus $G^{\prime}$ measured at very small strain amplitudes.

We have determined that the postshear properties of the suspension depend primarily on the strain amplitude employed in the preshear and not on the frequency or time of 
preshear. Dynamic preshear at moderate strain amplitudes $\left(\gamma_{0}=10 \%-100 \%\right)$ gives a much lower value for the recovered $G^{\prime}$ as compared to its initial level. This is postulated to be due to the rearrangement of flocs during the shearing process into larger flocs, thereby reducing the number of crosslinks in the network. On the other hand, during oscillatory shear at high strain amplitudes $(>300 \%)$, the network is broken down to flocs of small sizes and the network is able to build back almost to its initial level, after cessation of shear.

\section{ACKNOWLEDGMENTS}

We are grateful to Degussa Corporation for providing the fumed silica used in this study. We would also like to acknowledge the financial support of NACE International and the Department of Energy (DOE), Office of Basic Sciences for this project.

\section{References}

Aranguren, M. I., J. V. Degroot, E. Mora, and C. W. Macosko, "Effect of reinforcing fillers on the rheology of polymer melts," J. Rheol. 36, 1185-1182 (1992).

Cochrane, $H$. and C. S. Lin, "The influence of fumed silica properties on the processing, curing and reinforcement properties of silicone rubber," Rubber Chem. Technol, 66, 48-60 (1992).

de Rooij, R., "Rheology of weakly aggregating polystyrene latex dispersions," Ph.D. thesis, University of Twente, Netherlands, 1994.

Degussa Technical Bulletin No. 23, "AEROSIL as a thickening agent for liquid systems," Degussa Corp. 1989.

Degussa Technical Bulletin No. 6, "Hydrophobic AEROSIL: Manufacture, Properties and Applications," Degussa Corp., 1990.

Jones, D. A. R., B. Leary, and D. V. Boger, "The rheology of concentrated colloidal suspension of hard spheres." J. Colloid Interface Sci. 147, 479-495 (1991).

Kanai H., R. C. Navarrete, C. W. Macosko, and L. E. Scriven, "Fragile networks and rheology of concentrated suspensions," Rheol. Acta 31, 333-344 (1992).

Kanai, H. and T. Amari, "Strain-thickening transition in ferric oxide suspensions under oscillatory shear," Rheol. Acta 32, 539-549 (1993).

Khan, S. A., G. L. Baker, and S. Colson, "Composite polymer electrolytes using fumed silica fillers: Rheology and Ionic Conductivity," Chem. Mater. 6, 2359 - 2363 (1994).

Khan, S. A., M. A. Maruca, and I. M. Plitz, "Rheology of fumed silica dispersions for fiber-optic cables," Polym. Eng. Sci. 31, 1701-1707 (1991).

Khan, S. A., R. K. Prud'homme, E. M. Rabinovitch, and M. J. Sammon, "Rheology of the gelation of fluorimedoped silica sols," J. Non-cryst. Solids 110, 153-162 (1989).

Khan S. A. and N. J. Zoeller, "Dynamic rheological behaviour of flocculated fumed silica suspensions," J. Rheol. 37, 1225-1235 (1993).

Kosinski, L. E. and J. M. Caruthers, "Rheological properties of PDMS filled with fumed silica: II. Stress relaxation and stress growth," J. Non-Newt. Fluid Mech. 17, 69-89 (1985).

Mewis, J., and R. de Bleyser, "Dynamic behaviour of thixotropic systems," J. Colloid Interface Sci. 40. 360-369 (1972).

Mewis, J., "Thixotropy-A general review," J. Non-Newtonian Fluid Mech. 6, 1-20 (1979).

Nguyen, Q. D. and D. V. Boger, "Thixotropic behaviour of concentrated bauxite residue suspensions," Rheol. Acta 24, 427-437 (1985).

Schoukens, G. and J. Mewis, "Non-linear rheological behaviour and shear-dependent structure in colloidal dispersions." J. Rheol. 22, 381-394 (1978).

M. Van den Tempel, "Rheology of concentrated dispersions," J. Colloid Interface Sci. 71, 18-20 (1979).

Ziegelbaur, R. S. and J. M. Caruthers, "Rheological properties of PDMS filled with fumed silica: I. Hysteresis behaviour," J. Non-Newtonian Fluid Mech. 17, 45-68 (1985). 